\title{
Volatile Flavor Components in Green Tea Blended with Parched Naked Barley
}

Sung-Hee Choi*

\section{Department of Food Science and Nutrition, Blue-Bio Industry RIC, Dongeui-University, Busan 614-714, Korea}

Received May 15, 2012 /Revised June 20, 2012 / Accepted June 25, 2012

\begin{abstract}
To produce a new tea with a good flavor and functional properties using green tea of low quality, naked barley and barley were selected to blend with the green tea. The simultaneous distillation extraction method (SDE) using Likens and Nickerson's extraction apparatus was used to extract the volatile flavor compounds from the samples. The concentrated flavor extracts were analyzed and identified by GC and GC-MS. The GC patterns of the flavor components in two parched barleys were very different. The main volatile flavor components in two of the samples were alkyl pyrazines. Compounds including 3-methylbutanal, 2-methylbutanal, dihydro-2-methyl-3(2H)-furanone, 2,5-dimethyl pyrazine, and 3-ethyl-2.5-dimethyl pyrazine were isolated from the naked barley. Compounds including thiophenes, thiazoles, sulfides, and pyrroles with burnt odor were isolated from the barley. The parched naked barley was better than barley for adding to green tea. The main aroma components of the green tea blended with the naked barley were hexanol, hexanal, trans-2-hexenal, $\beta$ -ionone, a-ionone, alkyl pyrazines, 3-methylbutanal, 2-methylbutanal, and furfural.
\end{abstract}

Key words : Naked barley, barley, naked barley-green tea, alkyl pyrazines

\section{서 론}

우리나라 사람들은 녹차보다 향미가 강한 차제품을 선호하 고 있어 시판되고 있는 혼합녹차류 중에서는 구수한 숭늉 맛 을 내는 현미녹차의 소비도가 높다[4,6]. 본 연구는 상품성이 다소 떨어지는 중작 이하의 녹차를 효율적으로 이용하여 좋은 향미와 기능성을 가진 차를 제조하기 위하여 기능성 면에서도 다른 곡류에 비해 뒤떨어지지 않는 볶은 보리류를 이용하여 혼합녹차를 제조하고 차의 품질에 영향을 주는 중요한 인자 중의 하나인 향기성분을 분석한 것이다. 그 동안 볶은 곡류를 혼합한 녹차류의 향기연구로는 볶은 곡류 중 현미를 혼합한 현미녹차[4], 볶은 메밀을 혼합한 메밀녹차[7]를 제조하고 향 기 성분을 동정 한 바 있다. 또한, 새로운 기능성 곡류인 (주)제 주바이오머쉬가 자체 개발한 제조공법으로 생산되고 있는 상 황보리를 볶아서 녹차와 혼합한 상황보리녹차[8], 율무녹차 [11] 및 찰옥수수 혼합녹차[12] 등이 있어 이들 대부분의 혼합 녹차는 중작이하의 단독녹차보다는 관능적으로 선호도가 좋 은 것으로 나타났다. 본 연구에 사용된 쌀보리(裸麥 Naked barley: Hordeum vulgare L.)는 보리를 겉보리(대맥, covered barley, hulled barley)와 구분하여 부르는 명칭이다. 겉보리는 열매껍질이 씨에 달라붙어 쉽게 떨어지지 않고 쌀보리는 쉽게 떨어지는 것이 특징이다[15]. 겉보리를 도정한 것을 늘보리라 고 한다. 보리는 쌀 다음 가는 주곡으로 옛날부터 널리 이용되 어 온 주요 식량자원의 하나로서 관능적이며 영양학적인 기능

\footnotetext{
*Corresponding author

Tel : +82-51-890-1590, Fax : +82-51-890-2646

E-mail : choish@deu.ac.kr
}

성이 우수하다. 보리류는 덖으면 구수한 향을 갖게 되는데, 쌀보리와 늘보리를 같은 온도와 시간에서 볶으면 늘보리는 껍질이 빨리 타는 경향이 있고 뜨거운 물에 우리면 혼탁하여 혼합녹차용으로는 적합하지 않다는 것이 고찰되었다. 이에 본 연구에서는 먼저 볶은 보리와 쌀보리의 향기 성분을 비교하고 중급의 녹차에 볶은 쌀보리를 첨가하여 혼합한 쌀보리녹차를 제조하고 기호에 관계하는 향기성분을 분석하였다.

\section{재료 및 방법}

\section{실험재료}

쌀보리와 늘보리의 시료는 영천산을 시장에서 직접 구입하 여 각각 볶음기(Terada Co., Japan)를 사용하여 $140^{\circ} \mathrm{C}$ 에서 1시 간 볶은 것(Fig. 1)을 사용하였다. 쌀보리녹차는 쌀보리와 녹차 를 1: 1 로 혼합하여 사용하였고, 대조군으로는 볶은 쌀보리와 녹차를 각각 사용하였다. 녹차는 제주산 증제 중작 녹차를 사 용하였다.

\section{향기성분 농축물의 제조}

휘발성 향기성분의 추출에는 Likens and Nickerson형 동 시증류추출장치 $(\mathrm{SDE})$ 를 사용하였다. 즉, 각 시료를 분쇄하여 각 $50 \mathrm{~g}$ 과 증류수 $500 \mathrm{ml}$ 를 동시증류추출장치의 시료플라스 크에 넣고 용매플라스크에는 정제한 diethyl ether $50 \mathrm{ml}$ 와 비등석을 넣고, 1 시간 30 분 동안 가열 - 환류하면서 휘발성 성 분을 추출하였다. 추출 후 얻어진 에테르 추출물은 무수 황산 나트륨을 가해 하룻밤 탈수 후, 상압 $\left(38 \sim 40^{\circ} \mathrm{C}\right)$ 에서 diethyl ether를 제거, 농축한 후 휘발성성분 농축물을 얻었다. 


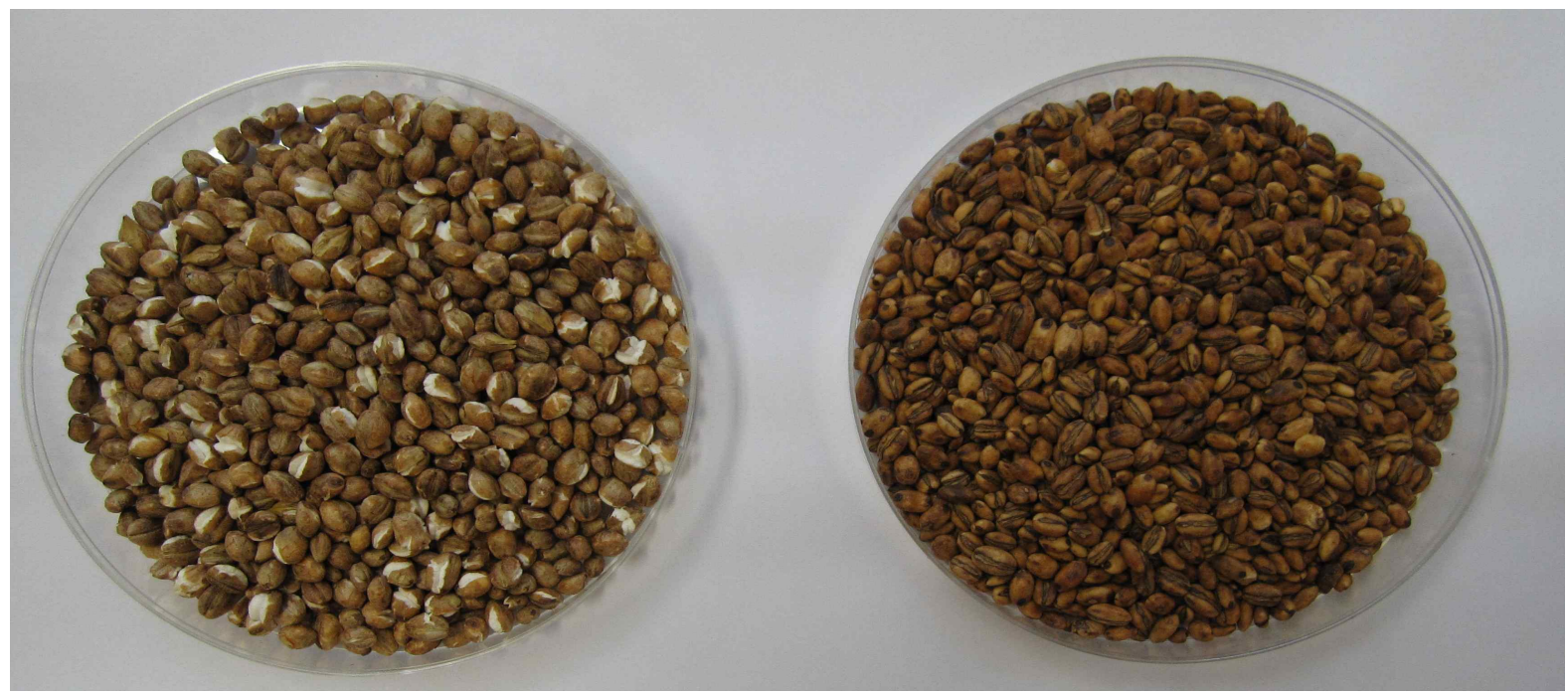

Fig. 1. Naked barley (left), Barley (right)

\section{향기성분의 분석 및 동정}

동시증류추출장치(SDE)에 의해 얻어진 휘발성 성분 농축 물은 Shimadzu GC-17A (Osaka, Japan) gas chromatography 에 의해 휘발성 성분을 분리하였다. 휘발성 성분의 검출에는 $\mathrm{FID}$ (불꽃이온화검출기), 칼럼은 HP-5MS capillary column $(30 \mathrm{~m} \times 0.25 \mathrm{~mm}$ i.d. $\times 0.25 \mu \mathrm{m}$ film thickness : J \& W Scientific, USA) 를 사용하였으며, 칼럼온도는 $50^{\circ} \mathrm{C}$ 에서 8 분간 유지시킨 후 $220^{\circ} \mathrm{C}$ 까지 $2^{\circ} \mathrm{C} / \mathrm{min}$ 의 속도로 승온 하였으며, GC 의 주입부는 $220^{\circ} \mathrm{C}$ 를 유지하였다. 운반기체로는 질소가스를 칼럼 내 유속은 $1 \mathrm{ml} / \mathrm{min}$ 으로 유지하였다. GC-MS 분석 장치 는 HP6890과 HP 5973 Mass Selective Detector 를 사용하고 detector의 온도는 $200^{\circ} \mathrm{C}$, mass range는 $25-450 \mathrm{~m} / \mathrm{z}$, linear velocity는 $40 \mathrm{~cm} / \mathrm{sec}$, multiplier voltage는 $1,500 \mathrm{~V}$, ionization voltage는 $70 \mathrm{eV}$ 로 설정하고, 운반기체로는 헬륨가스를 사용하였으며, 그 이외 칼럼의 온도를 비롯한 분석조건은 GC 의 분석조건과 동일하게 설정하였다. 휘발성성분의 동정은 mass spectral library data에 의한 검색, Wiley/NBS Registry of Mass Spectral Data, Eight Peak Index of Mass Spectra에 의한 문헌의 질량분석 데이터검색으로부터 물질을 추정하고, 표준물질의 머무름 시간 $\left(\mathrm{t}_{\mathrm{R}}\right)$ 의 일치에 의해 동정 하였다.

\section{결과 및 고찰}

\section{쌀보리와 늘보리의 휘발성 향기성분 비교}

두 시료의 휘발성 향기성분을 추출하고 농축한 농축물을 GC-MS로 분석 한 결과를 Table 1과 Table 2에 나타내었다. 가열에 의해 생성되는 휘발성화합물의 종류 중에는 달콤한 향을 띄는 반응중간체인 furfural 및 그 유도체 등이 있고 함질 소 heterocyclic 화합물인 pyrrole, pyrazine, 황과 질소를 포함
하는 heterocyclic 화합물인 황화합물들이 포함되어 있어 식 품의 독특한 가열향기에 기여하지만 구수한 향기에 주로 기여 하는 화합물은 alkyl pyrazine류이다. 가열과정 중에 생성되는 이러한 화합물들은 구수한 향을 내므로 대부분 바람직한 향이 라고 하지만 때로는 탄내나 쓴맛 등의 바람직하지 않는 향미 의 요소가 되기도 한다[7,8]. 이전의 연구에서 볶은 보리차의 향기성분으로 8종의 pyrazine류만 동정되고 있으나[15] 본 연 구에서는 두 종류에서 모두 구수한 향기에 기여하는 pyrazine 류가 28 종류가 분석되어 지금까지 연구한 다른 볶은 곡류에 비해서도 현저하게 많았다. 향기화합물의 종류는 늘보리에 많 았는데 늘보리에는 탄 냄새가 나는 pyrrole [1]이나 함황화합 물 중 좋지 않은 냄새에 영향을 미치는 thiophene이나 sulfide 류[11]와 같은 황화화합물의 종류와 함량이 더 많이 포함되어 있어 전체적으로 늘보리의 향미가 볶은 쌀보리보다 관능적으 로 기호도가 감소하는 요인으로 작용하는 것 같았다.

\section{쌀보리녹차의 휘발성 향기성분}

늘보리가 아니고 쌀보리를 혼합용녹차 재료로 사용한 큰 이유는 시료를 같은 온도와 시간에서 볶았을 때 늘보리는 껍 질이 빨리 타는 경향이 있었고 구수한 향미가 났지만 탄냄새 와 더불어 좋지 않은 냄새도 났다. 쌀보리는 적당히 구수한 향미가 났으며 뜨거운 물에 우려도 깨끗하게 우러나오고 늘보 리의 경우는 물에 우렸을 때 혼탁한 색깔을 나타내었다. 볶은 쌀보리를 녹차에 1:1로 혼합하여 쌀보리녹차를 제조하고 향기 성분을 동정하였다. 쌀보리녹차에서는 중급녹차와 볶은 쌀보 리에서 유래 되어진 것으로 보이는 많은 종류의 향기화합물이 분리되었다. 혼합녹차의 향기성분 화합물을 관능특성별로 묵 은 것을 Table 3 에 나타내었다. 볶은 쌀보리의 주요 휘발성 성분은 고소하고 볶은 향을 띄는 2,5-dimethyl pyrazine, 
Table 1. Volatile compounds identified in parched naked barley

\begin{tabular}{|c|c|c|c|}
\hline$t_{R}(\min )$ & Compounds & $t_{R}(\min )$ & Compounds \\
\hline 1.997 & Butanal & 2.037 & 2-Methyl furan \\
\hline 2.071 & Ethyl acetate & 2.185 & 2,3-Dihydro-3-methyl furan \\
\hline 2.339 & 3-Methyl butanal & 2.419 & 2-Methyl butanal \\
\hline 2.562 & 1-Penten-3-ol & 2.762 & 2-Ethyl furan \\
\hline 2.837 & 2.,5-Dimethyl furan & 2.917 & 2,6-Dimethyl furan \\
\hline 3.054 & 2-Vinyl furan & 3.163 & Pyrazine \\
\hline 3.237 & 2-Methyl-1-butanol & 3.265 & 4-Methyl-2-pentene \\
\hline 3.334 & 2-Methyl-2-butenal & 3.557 & 1H-Pyrrole \\
\hline 3.740 & Pentanol & 3.831 & cis-2-Penten-1-ol \\
\hline 4.140 & 2,3-dihydro-4-methyl furan & 4.248 & 2-Propyl furan \\
\hline 4.488 & Hexanal & 4.677 & Dihydro-2-methyl-3(2H)-Furanone \\
\hline 4.911 & 4-Methylthiazole & 5.134 & Methyl pyrazine \\
\hline 5.523 & Furfural & 5.792 & 1-Ethyl-1H-pyrrole \\
\hline 6.129 & 2-Methyl-1H-pyrrole & 6.255 & (E)-2-Hexenal \\
\hline 6.483 & Furfuryl alcohol & 7.032 & Hexanol \\
\hline 8.072 & 2-Heptanone & 8.404 & Nonanol \\
\hline 8.678 & Heptanal & 9.152 & 2,5-Dimethyl pyrazine \\
\hline 9.427 & Ethyl pyrazine & 9.604 & 2,3-Dimethyl pyrazine \\
\hline 10.255 & Ethenyl pyrazine & 12.159 & (E)-2-heptenal \\
\hline 12.250 & Benzaldehyde & 12.622 & 5-Methyl furfural \\
\hline 13.307 & Citronellol & 13.976 & 1-Octen-3-ol \\
\hline 14.639 & 2-Pentyl furan & 14.959 & 2-Ethyl-6-methyl pyrazine \\
\hline 15.154 & 2-Ethyl-5-methyl pyrazine & 15.256 & Trimethyl pyrazine \\
\hline 15.394 & 2-Ethyl-3-methyl pyrazine & 15.639 & Propyl pyrazine \\
\hline 15.982 & $(E, E)-2,4$-Heptadienal & 16.205 & 2-Ethenyl-6-methyl pyrazine \\
\hline 16.194 & 2-Methyl-6-vinyl pyrazine & 16.417 & Isopropyl pyranzine \\
\hline 18.280 & Phenyl acetaldehyde & 19.457 & Octenal \\
\hline 20.966 & 3-Ethyl-2,5-dimethyl pyrazine & 21.383 & 2-Ethyl-3,5-dimethyl pyrazine \\
\hline 21.549 & 2,6-Diethyl pyrazine & 21.709 & 2,5-Diethyl pyrazine \\
\hline 21.932 & 2-Methyl-5-propyl pyrazine & 22.086 & $(Z, E)-3,5$-Octadiene-2-one \\
\hline 22.366 & Dimethyl-2-vinyl pyrazine & 25.127 & 2-Methyl-3-(E)-propenyl pyrazine \\
\hline 26.562 & 2,3-Diethyl-5-methyl pyrazine & 26.710 & 3,5-Diethyl-2-methyl pyrazine \\
\hline 26.910 & 2,3,5-Trimethyl-6-ethyl pyrazine & 27.385 & 2,5-Diethyl-3-methyl pyrazine \\
\hline 28.493 & 1-(2-Furanyl methyl)-1H-pyrrole & 28.796 & 2-Acetyl pyrazine \\
\hline 29.842 & 3,5-Dimethyl-2-isobutyl pyrazine & 30.997 & 3-Phenyl furan \\
\hline 31.768 & 2,6-Diethyl-3,5-dimethyl pyrazine & 32.008 & 2,3-Diethyl-5,6-dimethyl pyrazine \\
\hline 33.152 & 2-Butyl-3-methyl pyrazine & 34.249 & 5-Pentyl-3H-furan-2-one \\
\hline 34.803 & 5-Ethoxy methyl furfural & 36.021 & Indole \\
\hline 36.221 & $(E, Z)$-2,4-Decadienal & 37.712 & $(E, E)$-2,4-Decadienal \\
\hline 39.364 & 5-Pentyl-2(3H)-furanone & 40.633 & 5-Heptyldihydro-2(3H)-furanone \\
\hline 42.011 & $\beta$-Damascenone & 42.353 & 2-Methyl-3-(1-methylethyl)-pyrazine \\
\hline 47.034 & $\beta$-Ionone & & \\
\hline
\end{tabular}

2,3-dimethyl- pyrazine, 3-ethyl-2,5-dimethyl pyrazine 등의 alkyl pyrazine 류와 달콤한 향을 띄는 3-methylbutanal, 2-methylbutanal, furfural 등이었다. 또한, 당류의 분해물로써 달콤한 향을 가지는 methyl butanal과 furfural, 5-hepthyl dihydro-2(3H)-furanone, 5-pentyl-2(3H)-furanone 등의 퓨란류 들이 많은 함량 동정되었다. 결과적으로 볶은 쌀보리의 냄새 는 구수한 pyrazine류의 향기 성분들과 아몬드 향으로 알려진 benzaldehyde나 pyrrole류 들이 구수한 향에 많은 기여를 하 는 것 같았다. 한편, 녹차의 주요 휘발성 성분[5,6]은 indole, phenyl ethanol 및 카로티노이드 색소분해물로 꽃향을 띄는 $\beta$-ionone 과 $a$-ionone 등의 함량이 많았다. Indole은 같은 야 부키타종의 경우라도 수확시기가 빠를수록 제조방법이 덖음 차 보다는 증제차에 많은 함량 들어 있다[2]. 이것은 재래종으 로 만든 녹차에는 그 함량이 적다[3]. 이는 희석된 농도에서는 
Table 2. Volatile compounds identified in parched barley

\begin{tabular}{|c|c|c|c|}
\hline$t_{R}(\min )$ & Compounds & $t_{R}(\min )$ & Compounds \\
\hline 2.020 & Ethyl acetate & 2.320 & 3-Methyl butanal \\
\hline 2.390 & 2-Methyl butanal & 2.550 & 1-Penten-3-ol \\
\hline 2.670 & 2,3-Pentanedione & 3.210 & Pyrazine \\
\hline 3.350 & Dimethyl disulfide & 3.480 & Pyrrole \\
\hline 3.840 & 3-Methyl thiophene & 4.550 & Hexanal \\
\hline 4.680 & 2-Methyl thiazole & 4.850 & 1-Ethyl-1H-pyrrole \\
\hline 5.010 & 4-Methyl thiazole & 5.330 & Methyl pyrazine \\
\hline 5.920 & Furfural & 6.230 & 5-Methyl thiazole \\
\hline 6.450 & 2-(2-Propenyl)-furan & 6.540 & Fufuryl alcohol \\
\hline 6.680 & Ethyl benzene & 6.740 & 2-Methyl thiophene \\
\hline 6.880 & 2,4-Dimethyl thiophene & 7.210 & Hexenol \\
\hline 7.480 & 2,3-Dimethyl thiophene & 7.900 & 2,4-Dimethyl thiazole \\
\hline 8.250 & 2-Heptanone & 8.470 & 2-Ethyl thiazole \\
\hline 8.960 & Ethenyl thiophene & 9.280 & $\underline{4,5 \text {-Dimethyl isothiazole }}$ \\
\hline 9.460 & 2-Acethyl furan & 9.640 & 2,5-Dimethyl pyrazine \\
\hline 9.850 & Ethyl pyrazine & 9.960 & 2,3-Dimethyl pyrazine \\
\hline 10.740 & Ethenyl pyrazine & 11.110 & 2-Ethyl-4-methyl thiophene \\
\hline 11.510 & 5-Ethyl thiazole & 11.670 & 1-butyl-1H-pyrrole \\
\hline 11.810 & Camphene & 12.120 & Propyl benzene \\
\hline 12.260 & 2-Butyl furan & 12.650 & $(E)$-2-heptanal \\
\hline 13.010 & 5-Methyl furfural & 13.160 & Dimethyl trisulfide \\
\hline 13.520 & 2-Ethyl-4-methyl thiazole & 14.500 & 1-Octen-3-ol \\
\hline 14.660 & 3-Ethyl-2,4-dimethyl-1H-pyrrole & 14.940 & 2-Pentyl furan \\
\hline 15.060 & Benzo furan & 15.520 & 2-Ethyl-6-methyl pyrazine \\
\hline 15.760 & 2-Ethyl-5-methyl pyrazine & 15.870 & 2-Ethyl-3-methyl pyrazine \\
\hline 16.090 & 4-Ethyl-2-methyl pyrrole & 16.250 & propyl pyrazine \\
\hline 16.460 & $\underline{\text { 5-Ethyl-4-methyl thiazole }}$ & 16.830 & 2-Ethenyl-6-methyl pyrazine \\
\hline 17.650 & limonene & 18.840 & Phenyl acetaldehyde \\
\hline 19.360 & 1-(2-Methylbutyl)-pyrrole & 19.610 & Butyl benzene \\
\hline 19.830 & $\delta$-Terpinene & 20.160 & (2-Methyl propyl)-pyrazine \\
\hline 20.620 & Tolualdehyde & 21.360 & 3-Ethyl-2,5-dimethyl pyrazine \\
\hline 21.650 & 2-Furfuryl furan & 21.870 & 2-Ethyl-3,5-dimethyl pyrazine \\
\hline 21.970 & 2,6-Dimethyl pyrazine & 22.100 & 2,5-Dimethyl pyrazine \\
\hline 22.830 & 2-Methyl-5-propyl pyrazine & 25.450 & 3,5-Dimethyl-1,2,4-trithiazolane \\
\hline 25.550 & 2-Methyl-3-propyl pyrazine & 25.830 & 2-Isopropyl-4-methyl thiazole \\
\hline 26.850 & 2,3-Dimethyl-5-methyl pyrazine & 27.070 & 3,5-Diethyl-2-methyl pyrazine \\
\hline 27.270 & 2,3,5-Trimethyl-6-ethyl pyrazine & 27.360 & 2,5-Diethyl-3-propyl pyrazine \\
\hline 27.640 & 2-Nonenal & 28.480 & 2,3-Dimethyl-5-(2-propenyl)-pyrazine \\
\hline 28.830 & Fufuryl pyrrole & 29.200 & 3-Ethyl-2-formyl thiophene \\
\hline 30.310 & 3,5-Dimethyl-2-isobutyl pyrazine & 30.830 & Fufuryl methyl disulfide \\
\hline 31.140 & 2,3-Dimethyl-5-(2-methyl propyl)-pyrazine & 31.740 & 3-Phenyl furan \\
\hline 33.700 & 2-Isopentyl-3-methyl pyrazine & 34.390 & 2-Isoamyl-6-methyl pyrazine \\
\hline 35.550 & Phentyl thiophene & 35.870 & 1-(2-Methyl butyl)-2-formyl pyrrole \\
\hline 37.880 & 2-Methoxy-4-vinyl phenol & 38.200 & 2,5-Dimetjyl-3-(2-methyl butyl)pyrazine \\
\hline 38.660 & $(E, E)$-2,4-decadienal & 44.270 & 3-Phenyl thiophene \\
\hline
\end{tabular}

재스민계의 꽃향을 띄며 차향기 전체에 조화를 이루도록 하며 보향효과도 있다[1,2]. 풋풋한 풀 냄새를 띄는 $\mathrm{C}_{6}$ alcohol 류와 $\mathrm{C}_{6}$ aldehyde 류인 hexanol, hexanal 및 trans-2-hexenal 등도 다소 들어 있었다. 녹차의 향기 성분으로 구수한 향기에 기여 하는 alkyl pyrazine 류가 동정되지 않은 것은 이 연구에 사용
한 녹차는 덖음차가 아니고 증제녹차이며 덖음차라 할지라도 지나치게 덖지 않는 한 alkyl pyrazine 류의 함량은 극히 적다 [7]. 복은 쌀보리를 혼합한 녹차의 경우, 관능적으로 녹차의 특유한 향에 우리나라사람들이 좋아하는 구수한 향이 부가되 어 바람직한 향으로 되었다. 볶은 쌀보리 혼합 녹차의 경우 
Table 3. Proportion of characteristic aroma compounds in green tea, naked barley, and naked barley-green tea

\begin{tabular}{|c|c|c|c|c|c|}
\hline \multirow{2}{*}{$\begin{array}{c}\text { Aroma } \\
\text { compound }\end{array}$} & \multirow{2}{*}{$\begin{array}{c}t_{R} \\
(\mathrm{~min})\end{array}$} & \multirow{2}{*}{ Compounds } & \multicolumn{3}{|c|}{ Peak area (\%) } \\
\hline & & & $G^{1)}$ & $\mathrm{NB}^{2)}$ & $\mathrm{NBG}^{3)}$ \\
\hline \multicolumn{6}{|l|}{ Floral } \\
\hline & 33.001 & Benzyl alcohol & 1.50 & & 0.08 \\
\hline & 33.542 & Phenyl acetaldehyde & 0.52 & 1.91 & 0.88 \\
\hline & 35.529 & Acetophenone & 0.50 & & 0.18 \\
\hline & 39.221 & Phenyl ethanol & 1.65 & & 0.52 \\
\hline & 54.630 & Indole & 18.41 & 0.03 & 11.44 \\
\hline & 61.006 & cis-Jasmone & 3.32 & & 1.11 \\
\hline & 63.635 & $a$-Ionone & 2.49 & & 0.70 \\
\hline & 63.849 & Dihydro- $\beta$-Ionone & 0.15 & & 0.07 \\
\hline & 73.486 & $\beta$-Ionone & 28.93 & & 7.89 \\
\hline \multirow{2}{*}{\multicolumn{6}{|c|}{ Sweet, Fruity }} \\
\hline & & & & & \\
\hline & 8.199 & 3-Methyl butanal & 0.93 & 7.22 & 0.80 \\
\hline & 8.482 & 2-Methyl butanal & 0.10 & 6.00 & 0.62 \\
\hline & 10.665 & 2-Ethyl furan & 1.50 & 0.63 & 0.15 \\
\hline & 14.109 & Pentanol & & 2.86 & 1.02 \\
\hline & 14.665 & 2,3-Dihydro-4-methyl furan & 1.98 & 0.03 & 0.12 \\
\hline & 15.733 & Dihydro-2-methyl-3(2H)-furanone & & 1.40 & 0.18 \\
\hline & 16.474 & Furfural & 0.50 & 1.54 & 1.04 \\
\hline & 18.707 & Furfuryl alcohol & & 0.32 & 0.43 \\
\hline & 20.449 & 2-Heptanone & 0.08 & 1.23 & 0.13 \\
\hline & 21.456 & Heptanal & 2.80 & 0.36 & 0.98 \\
\hline & 26.708 & 5-Methyl furfural & & 0.48 & 0.19 \\
\hline & 32.550 & Limonene & 0.16 & & 0.21 \\
\hline & 45.976 & Safranal & 0.10 & & 0.55 \\
\hline & & & 8.15 & 22.07 & 6.42 \\
\hline \multicolumn{6}{|c|}{ Green, Grassy } \\
\hline & 15.707 & Hexanal & 1.80 & 4.90 & 1.96 \\
\hline & 18.147 & trans-2-Hexenal & 0.28 & 0.20 & 0.06 \\
\hline & 19.402 & Hexanol & 1.07 & 0.94 & 0.66 \\
\hline & 59.913 & 3-Hexenyl hexanoate & 1.98 & & 0.59 \\
\hline & & & 5.13 & 6.04 & 3.27 \\
\hline \multicolumn{6}{|l|}{ Roasty } \\
\hline & 12.284 & Pyrazine & & 1.40 & 0.86 \\
\hline & 12.813 & 1H-Pyrrole & & 3.06 & 0.57 \\
\hline & 15.976 & Methyl pyrazine & & 2.16 & 0.93 \\
\hline & 22.730 & 2,5-Dimethyl pyrazine & & 11.75 & 6.88 \\
\hline & 22.940 & Ethyl pyrazine & & 0.46 & 0.42 \\
\hline & 23.105 & 2,3-Dimethyl pyrazine & & 0.12 & 0.34 \\
\hline & 23.756 & Ethenyl pyrazine & & 0.02 & 0.18 \\
\hline & 26.427 & Benzaldehyde & & 1.33 & 0.74 \\
\hline & 29.916 & 2-Ethyl-6-methyl pyrazine & & 3.19 & 2.93 \\
\hline & 30.166 & 2-Ethyl-5-methyl pyrazine & & 1.67 & 1.31 \\
\hline & 30.366 & 2-Ethyl-3-methyl pyrazine & & 2.14 & 1.26 \\
\hline & 30.661 & Propyl pyrazine & & 0.25 & 0.16 \\
\hline & 31.406 & 2-Ethenyl-6-methyl pyrazine & & 0.18 & 0.34 \\
\hline & 31.939 & 2-Methyl-6-vinyl pyrazine & & 0.40 & 0.08 \\
\hline & 32.277 & 3-Ethyl-1H-pyrrole & & 0.23 & 0.09 \\
\hline & 36.544 & 3-Ethyl-2,5-dimethyl pyrazine & & 3.55 & 1.56 \\
\hline & 36.657 & 2-Ethyl-3,5-dimethyl pyrazine & & 0.22 & 0.63 \\
\hline & 36.718 & 2,6-dimethyl pyrazine & & 0.22 & 0.48 \\
\hline & 36.790 & 2,5-dimethyl pyrazine & & 0.50 & 0.56 \\
\hline & 37.637 & Dimethyl-2-vinyl pyrazine & & 0.10 & 0.32 \\
\hline & 41.115 & 2-Methyl-5-propyl pyrazine & & 0.09 & 0.08 \\
\hline & 41.349 & 5H-5-Methyl-6,7-dihydropenta pyrazine & & 0.18 & 0.06 \\
\hline & 42.850 & 2,3-Diethyl-5-methyl pyrazine & & 0.65 & 0.35 \\
\hline & 43.025 & 3,5-Diethyl-2-methyl pyrazine & & 0.21 & 0.25 \\
\hline & 43.243 & 2,3,5-Trimethyl-6-ethyl pyrazine & & 0.20 & 0.25 \\
\hline & 44.474 & 1-2(-Furanyl methyl)-1H-pyrrole & & 0.28 & 0.78 \\
\hline & 46.507 & 3,5-Dimethyl-2-isobutyl pyrazine & & 0.14 & 0.42 \\
\hline & 48.735 & 2-Butyl-3-methyl pyrazine & & 0.21 & 0.41 \\
\hline & & & 0 & 34.91 & 23.24 \\
\hline
\end{tabular}

${ }^{1)} \mathrm{G}$ : Green tea

${ }^{2)} \mathrm{NB}$ : Naked barley

${ }^{3)} \mathrm{NBG}$ : Naked barley-green tea 
향기 성분으로 쌀보리에서 유래하는 alkyl pyrazine 류와 녹차 류에서 유래되는 꽃향 성분들의 peak area는 적절하게 균형을 이루어 나타났다. 결론적으로 중급녹차에 볶은 쌀보리를 혼합 하여 제조할 시, 볶은 쌀보리의 구수한 향과 맛이 녹차와 잘 조화되어 우리나라 사람들의 기호에 잘 부합할 뿐 만 아니라, 녹차 음용의 초보자적 사람들의 기호에 적합하여, 중급녹차의 대중화에 대한 기여도가 높을 것으로 생각되어졌다.

\section{감사의 글}

본 연구는 2011년도 지식경제부 부산광역시. 지원 지역혁신 센터사업(RIC) 동의대학교 블루바이오 소재 개발 및 실용화 지원 센터의 지원으로 이루어 졌으며 이에 감사드립니다.

\section{References}

1. Akahoshi, G. 1983. Kouryonokagaku. pp. 295-298, Dainihontosyo. Tokyo, Japan.

2. Choi, S. H. 1991. Studies on flavor components of commercial Korean green teas. Korean J. Food Sci. Technol. 33, 98-101.

3. Choi, S. H. and Bae, J. E. 1996. The aroma components of green tea, the products of Mt. Chiri garden. J. Korean Soc. Food Nutr. 25, 478-483.
4. Choi, S. H. and Lee, D. H. 1997. Aroma compounds of bran rice-green tea. J. Korean Tea Soc. 3, 37-45.

5. Choi, S. H. 2001. Volatile aroma compounds of Korean semi-fermented teas. Korean J. Food Sci. Technol. 33, 529-533.

6. Choi, S. H. 2002. Korean tea and world tea, pp. 37-37, Jungang Life Publishing. Seoul, Korea.

7. Choi, S. H. 2007. Volatile flavor components of buckwheat-green tea. J. Life Sci. 17,1111-1114.

8. Choi, S. H. and K. T. Chung. 2009. Volatile flavor components in a mixed tea of phellinus linteus-barley and green tea. J. Korea Tea Soc. 15, 107-110.

9. Choi, S. H. 2010. Effects on pyrazines, reaction products of maillard reaction in flavor of green tea and substitution tea.

10. Fujimaki, M., Abe, D., Hayashi, G. and Arai, S. 1982. pp. 395-395, Koryonogiten, Asakurasyoten, Tokyo, Japan.

11. Jeon, J. Y. and Choi, S. H. 2009. Volatile flavor components in a mixed tea of coix lachryma-jobi L. var. mayuen stapf and green tea. J. Korea Tea Soc. 15, 69-75.

12. Jeon, J. Y. and Choi, S. H. 2011. Volatile flavor components in parched waxy corns (zea mays L.) tea. J. Korea Tea Soc. $17,83-86$.

13. Lee, Y. C. 1997. Change of food flavor in manufacturing process. Food Sci. Ind 30, 10-25.

14. Lee, Y. E. and Hong, S. H. 2003. Medicinal food materials. pp. 36-39, Kyomoonsa. Seoul, Korea.

15. Yamanishi, T. 1989. Aroma of semi-fermented tea Goryo (in Japanese) No. 161. 61

\section{초록 : 볶은 쌀보리를 혼합한 녹차의 휘발성 향기성분}

\section{최성희*}

(동의대학교 식품영양학과 . 블루바이오 소재개발 센터)

중작녹차를 효율적으로 이용하여 좋은 향미와 기능성을 가진 차를 만들기 위해 덖은 쌀보리에 중작녹차를 혼 합하였다. 쌀보리녹차의 휘발성 향기성분의 추출은 Likens and Nickerson형 추출 장치를 사용한 동시증류추출방 법에 의해 완수였으며, 그 농축된 추출물은 GC (Gas chromatography)와 GC-MS (Gas chromatography - mass spectrometry)에 의해 분석되었다. 3-methylbutanal, 2-methylbutanal, 그리고 2,5-dimethyl pyrazine 등의 화합물 이 쌀보리로부터 분리, 동정되었다. 그리고 녹차에서는 a-terpinolene, indole, $\beta$-ionone 등을 포함한 화합물을 분리, 동정하였으며, 또한 쌀보리와 녹차를 혼합한 쌀보리녹차에서는 2,5-dimethyl pyrazine, indole, 3-ethyl-2,5-dimethyl pyrazine 등을 포함한 화합물들이 분리, 동정되었다. 그 결과로부터 일반 중작녹차보다 볶은 쌀보 리와 녹차를 혼합한 쌀보리녹차가 관능적으로 더 좋은 향미를 주는 요인으로 작용하는 것 같았다. 